TRANSACTIONS OF THE

AMERICAN MATHEMATICAL SOCIETY

Volume 362, Number 9, September 2010, Pages 4853-4869

S 0002-9947(10)05171-8

Article electronically published on April 28, 2010

\title{
THE CAUCHY PROBLEM FOR $p$-EVOLUTION EQUATIONS
}

\author{
MASSIMO CICOGNANI AND FERRUCCIO COLOMBINI
}

\begin{abstract}
In this paper we deal with the Cauchy problem for evolution equations with real characteristics. We show that the problem is well-posed in Sobolev spaces assuming a suitable decay of the coefficients as the space variable $x \rightarrow \infty$. In some cases, such a decay may also compensate a lack of regularity with respect to the time variable $t$.
\end{abstract}

\section{IntRoduction AND MAIN RESUlts}

Let us consider the Cauchy problem in $[0, T] \times \mathbb{R}_{x}$,

$$
\left\{\begin{array}{l}
L u=0 \\
u(0, x)=u_{0}, \partial_{t} u(0, x)=u_{1}
\end{array}\right.
$$

for the operator

$$
L:=D_{t}^{2}+2 b_{p}(t) D_{t} D_{x}^{p}+a_{2 p}(t) D_{x}^{2 p}+\sum_{j=0}^{p-1} b_{j}(t, x) D_{t} D_{x}^{j}+\sum_{k=0}^{2 p-1} a_{k}(t, x) D_{x}^{k},
$$

where $p \geq 2$ is a positive integer, $D=\frac{1}{i} \partial$. For the coefficients $a_{k}, k<2 p$, and $b_{j}$, $j<p$, in general complex valued functions, we assume

$$
a_{k}, b_{j} \in C\left([0, T] ; \mathcal{B}^{\infty}\right), k=0, \ldots, 2 p-1, j=0, \ldots, p-1,
$$

where $\mathcal{B}^{\infty}=\mathcal{B}^{\infty}\left(\mathbb{R}_{x}\right)$ denotes the space of all functions $f(x)$ which are bounded in $\mathbb{R}_{x}$ together with all their derivatives. The leading coefficients $a_{2 p}$ and $b_{p}$ are assumed to be real valued continuous functions such that

$$
\Delta(t):=b_{p}^{2}(t)-a_{2 p}(t) \geq \lambda_{0}>0 ;
$$

hence $L$ is a $p$-evolution operator with real distinct characteristic roots $\tau=$ $\left(-b_{p}(t) \pm \sqrt{\Delta(t)}\right) \xi^{p}$. In view of the Lax-Mizohata theorem, for any $p \geq 1$, to have real roots is a necessary condition in order to solve uniquely (1.1) in Sobolev spaces in a neighborhood of $t=0,9$. As far as applications are concerned, for $p=2$ and $b_{p}=0$ we have a vibrating beam equation. Still for $p=2$, the related first order (in time) equation $L_{1} u=0$,

$$
L_{1}:=D_{t}+e_{p}(t) D_{x}^{p}+\sum_{j=0}^{p-1} e_{j}(t, x) D_{x}^{j}, e_{p}(t) \in \mathbb{R},
$$

Received by the editors February 17, 2009.

2010 Mathematics Subject Classification. Primary 35G10, 35L15.

Key words and phrases. Evolution equations with real characteristics.

(C)2010 American Mathematical Society 
is a Schrödinger equation, while for $p=3$, the principal part of $L_{1} u=0$ is the same as in the Korteweg-De Vries equation.

The kovalevskian case $p=1$ in (1.2) is that of a strictly hyperbolic operator. Just in this latest case, by well-known results starting from [3, we know that there is a strict and deep relation between the well-posedness of the Cauchy problem (1.1) and the modulus of Hölder continuity of the coefficients with respect to the time variable. For $p \geq 2$ these topics have been studied in 11. In particular, from the results we have obtained there, we have the following theorem:

Theorem 1.1 ([1]). Besides (1.3) and (1.4), let us assume

$$
a_{j+p}=a_{j+p}(t), b_{j}=b_{j}(t), a_{j+p}, b_{j} \in C^{0, j / p}([0, T] ; \mathbb{R}), \quad j=1, \ldots, p .
$$

Then, for every choice of Cauchy data $u_{0} \in H^{s}, u_{1} \in H^{s-p}$ the problem (1.1) has a unique solution $u \in C\left([0, T] ; H^{s}\right) \cap C^{1}\left([0, T] ; H^{s-p}\right)$.

When the conclusions of Theorem 1.1 hold, we say that the problem (1.1) is well-posed in the Sobolev space $H^{s}$. We notice that the leading coefficients $a_{2 p}$ and $b_{p}$ are Lipschitz continuous in (1.6).

Remark 1.2. In view of the counterexamples we showed in [1] and [2], the regularity given by (1.6) is the optimal one for the well-posedness in $H^{s}$.

Remark 1.3 ([1). If one weakens the hypothesis (1.6) by assuming

$$
\begin{gathered}
a_{j+p}=a_{j+p}(t) \in \mathbb{R}, \sup _{t_{1} \neq t_{2}} \frac{\left|a_{j+p}\left(t_{1}\right)-a_{j+p}\left(t_{2}\right)\right|}{\left|t_{1}-t_{2}\right|^{j / p}|\log | t_{1}-t_{2}||}<+\infty, \\
b_{j}=b_{j}(t) \in \mathbb{R}, \sup _{t_{1} \neq t_{2}} \frac{\left|b_{j}\left(t_{1}\right)-b_{j}\left(t_{2}\right)\right|}{\left|t_{1}-t_{2}\right|^{j / p}|\log | t_{1}-t_{2}||}<+\infty, \\
\quad j=1, \ldots, p,
\end{gathered}
$$

then there exists $\delta>0$ such that for every choice of Cauchy data $u_{0} \in H^{s}, u_{1} \in$ $H^{s-p}$, the problem (1.1) has a unique solution $u \in C\left([0, T] ; H^{s-\delta}\right) \cap C^{1}([0, T]$; $\left.H^{s-p-\delta}\right)$. In this case we say that the problem is well-posed in $H^{\infty}$ with a loss of derivatives. Suitable counterexamples in [1] and [2] show that the assumption (1.7) is the optimal one for the well-posedness of (1.1) in $H^{\infty}$. We notice that the leading coefficients $a_{2 p}, b_{p}$ have the so-called log-Lipschitz regularity in (1.7).

Remark 1.4 ([1]). If one has

$$
\begin{aligned}
a_{j+p}=a_{j+p}(t), a_{j+p} \in C^{0, \alpha_{j+p}}([0, T] ; \mathbb{R}), \\
\quad b_{j}=b_{j}(t), b_{j} \in C^{0, \beta_{j}}([0, T] ; \mathbb{R}), j=1, \ldots, p,
\end{aligned}
$$

with $\alpha_{j+p}<j / p$ or $\beta_{j}<j / p$, even for a single coefficient $a_{j+p}$ or $b_{j}$, then the problem (1.1) may in general be well-posed only in Gevrey spaces.

Remark 1.5. In the limit hyperbolic case $p=1$, the results of 1 that we recall here in Remark 1.3 are in line with the well-known results of [3] for coefficients depending only on the variable $t$ and of 4 for the equation

$$
u_{t t}-\partial_{x}\left(a(t, x) u_{x}\right)=0
$$

with a $\log$-Lipschitz coefficient $a(t, x)$ with respect to both variables $(t, x)$. See also [5] for the study of a general second order hyperbolic operator with log-Lipschitz coefficients. 
After the sharp regularity in $t$ for the well-posedness in Sobolev spaces has been established with real coefficients $a_{p+j}(t), b_{j}(t), 1 \leq j \leq p-1$, some natural questions arise looking at the results above. The first question is which coefficients $a_{k}$ with $k>p$ and $b_{j}$ with $j>0$ may also depend on the space variable $x$, and, together with this question, we have the following two:

- Question A:

Can the coefficients $a_{k}$ with $p<k<2 p$ and $b_{j}$ with $0<j<p$ be complex valued as those coefficients with $k \leq p$ and $j=0$ are?

- Question B:

How could we compensate for a lack of Hölder continuity $\alpha_{k}<(k-p) / p$ or $\beta_{j}<j / p$ of a coefficient $a_{k}$ or $b_{j}$ ?

For instance, the necessity of a positive answer to the first problem arises in a natural way in the Euler-Bernoulli model of the vibrating beam, which corresponds to $p=2$ and $b_{p}=0$ in (1.1) and (1.2). In this case, $u=u(t, x)$ represents the displacement, and the shear force is proportional to $\partial_{x}^{3} u=-i D_{x}^{3} u$, so that one has to deal with a complex coefficient of $D_{x}^{3}$ in (1.2).

Actually, we cannot give positive answers to A or B if the coefficients do not depend on $x$, since a suitable decay is needed as $|x| \rightarrow+\infty$. In fact, already for a first order operator $L_{1}$ as in (1.5) one needs decay assumptions at least on the imaginary parts $e_{j}^{\prime \prime}$ of the coefficients $e_{j}=e_{j}^{\prime}+i e_{j}^{\prime \prime}, e_{j}^{\prime}, e_{j}^{\prime \prime} \in \mathbb{R}$. Precisely, denoting hereafter $\langle x\rangle$ for $\sqrt{1+x^{2}}$, one has to assume

$$
\left|e_{j}^{\prime \prime}(t, x)\right| \leq C\langle x\rangle^{-j /(p-1)}, j=1, \ldots, p-1 ;
$$

see, for example, [7 for $p=2$ and Section 2 of this paper for $p \geq 2$. Indeed, in the Schrödinger case $p=2$, the necessity of the condition (1.8) for the well-posedness in $H^{\infty}$ has been fully proved; see, e.g., 6]. Still for $p=2$, one needs the stronger condition

$$
\left|e_{p-1}^{\prime \prime}(t, x)\right| \leq C\langle x\rangle^{-\sigma}, \sigma>1,
$$

for the $H^{s}$ well-posedness.

We state our results concerning these questions in the main two cases $p=2$ and $p=3$. We could give similar results for general $p$ but the number of cases that one has to consider grows very fast with $p$, particularly for Question B. Besides (1.4), we also need to assume that the characteristic roots do not vanish for $\xi \neq 0$, that is,

$$
\left|a_{2 p}(t)\right| \geq \lambda_{1}>0 .
$$

First we state our results for Question A, assuming the sharp Hölder continuity of the coefficients:

Theorem 1.6. Let us consider the problem (1.1) in the case $1<p \leq 3$ under the assumptions (1.4), (1.9), (1.3). Let the leading coefficients $a_{2 p}(t), b_{p}(t)$ be Lipschitz continuous functions and

$$
a_{p+j}, b_{j} \in C^{0, j / p}\left([0, T] ; \mathcal{B}^{\infty}\right), 1 \leq j \leq p-1 .
$$

Let us write $a_{k}=a_{k}^{\prime}+i a_{k}^{\prime \prime}, b_{j}=b_{j}^{\prime}+i b_{j}^{\prime \prime}$, with real $a_{k}^{\prime}, a_{k}^{\prime \prime}, b_{j}^{\prime}, b_{j}^{\prime \prime}$, and let us assume that

$$
\left|a_{2 p-1}^{\prime \prime}\right|+\left|b_{p-1}^{\prime \prime}\right| \leq C_{0}\langle x\rangle^{-\sigma}, \sigma \geq 1
$$


and, for $p>2$, that

$$
\left|\partial_{x} a_{2 p-1}^{\prime}\right|+\left|\partial_{x} b_{p-1}^{\prime}\right|+\left|a_{2 p-2}^{\prime \prime}\right|+\left|b_{p-2}^{\prime \prime}\right| \leq C\langle x\rangle^{-(p-2) /(p-1)} .
$$

Then the Cauchy problem (1.1) is well-posed in:

$H^{s}$ for $\sigma>1$ in (1.11);

$H^{\infty}$ with a loss of derivatives for $\sigma=1$ in (1.11), provided that (1.9) is specified by

$(1.9)_{+} \quad a_{2 p}(t) \geq \lambda_{1}>0$.

Remark 1.7. The condition (1.9) + means that the two characteristic roots have the same sign at any $t \in[0, T]$ and $\xi \neq 0$. In Theorem 1.10 , we deal with the remaining case of characteristic roots of different signs and $\sigma=1$ in (1.11).

It is also interesting to point out that under the assumption (1.9) + , Hölder continuity is required only for the real parts of the coefficients. On the other hand, for $p>2$, we have to assume some extra decay conditions for the derivatives in $x$ of the imaginary parts in order to allow them to be merely continuous functions of $t$. In fact, we have:

Proposition 1.8. Let (1.9) be fulfilled in the case (1.9)+. Maintaining all the other assumptions, the conclusions of Theorem 1.6 hold true, weakening (1.10) into

(1.10) $\Re \quad a_{p+j}^{\prime}, b_{j}^{\prime} \in C^{0, j / p}\left([0, T] ; \mathcal{B}^{\infty}\right), 1 \leq j \leq p-1$,

provided that, for $p>2$,

(1.12) $)^{\prime} \quad\left|\partial_{x} a_{2 p-1}^{\prime \prime}\right|+\left|\partial_{x} b_{p-1}^{\prime \prime}\right|+\left|\partial_{x x} a_{2 p-1}^{\prime \prime}\right|+\left|\partial_{x x} b_{p-1}^{\prime \prime}\right| \leq C\langle x\rangle^{-(p-2) /(p-1)}$

is added to (1.12) and that the further condition

$$
\left|\partial_{x x x} a_{2 p-1}^{\prime \prime}\right|+\left|\partial_{x x x} b_{p-1}^{\prime \prime}\right| \leq C\langle x\rangle^{-(p-2-1 / 2) /(p-1)}
$$

is assumed.

Remark 1.9. If in Theorem 1.6 or Proposition 1.8 the coefficients satisfy (1.7), uniformly with respect to the variable $x$, instead of (1.10) or (1.10) for $j<p$, then the problem (1.1) is well-posed in $H^{\infty}$ with a loss of derivatives for any $\sigma \geq 1$ in (1.11). This time, a loss comes from the logarithm in the modulus of continuity with respect to $t$, independently of the behaviour for $|x| \rightarrow \infty$; cf. Remark 1.3 .

The set of conditions (1.4), (1.9) + does not allow us to consider the case $b_{p}(t)=0$. If (1.9) is satisfied in the opposite case,

(1.9) -

$$
-a_{2 p}(t) \geq \lambda_{1}>0
$$

then (1.4) is fulfilled for any coefficient $b_{p}(t)$, but now we need more than Hölder regularity with respect to the time variable of the coefficients in dealing with the case $\sigma=1$ in (1.11). It seems that for characteristic roots of opposite signs, the loss of derivatives coming from $\sigma=1$ has to be compensated for by a higher regularity in $t$ of the coefficients. In fact, we have:

Theorem 1.10. Let us consider the problem (1.1) in the case $1<p \leq 3$ under the assumptions (1.3), (1.9) . Assume that (1.11) is satisfied with $\sigma=1$ and, for $p>2$, assume also (1.12). Let us denote

$$
N_{0}:=\frac{C_{0}}{p}\left(\frac{1}{\sqrt{\lambda_{0}}}+\frac{2}{\lambda_{1}}\right)
$$

with $C_{0}, \lambda_{0}$ and $\lambda_{1}$ the constants in (1.11), (1.4) and (1.9), respectively. 
If the coefficients are such that

$$
\begin{aligned}
& a_{2 p}, b_{p} \in C^{N+p}\left([0, T] ; \mathbb{R}_{+}\right), \\
& \quad a_{k} \in C^{N+k-p}\left([0, T] ; \mathcal{B}^{\infty}\right), \quad b_{j} \in C^{N+j}\left([0, T] ; \mathcal{B}^{\infty}\right), \\
& \quad 0 \leq k \leq 2 p-1,0 \leq j \leq p-1,
\end{aligned}
$$

for $N \geq N_{0}$, then the Cauchy problem (1.1) is well-posed in $H^{\infty}$ with a loss of $N_{0}$ derivatives.

Passing to Question B, Proposition 1.8 says that the regularity in $t$ and the behaviour for $|x| \rightarrow+\infty$ are essentially independent for the imaginary parts of the coefficients, at least in the case of characteristic roots of the same sign. On the other hand, from Theorem 1.6 we do not need any condition as $|x| \rightarrow+\infty$ for real parts which have the sharp Hölder regularity. Now we state for the real parts $a_{p+j}^{\prime}$, $b_{j}^{\prime}, j=1, \ldots, p-1$, a compensation between the regularity in $t$ and the decay as $|x| \rightarrow+\infty$ which cannot take place for the imaginary parts $a_{k}^{\prime \prime}, b_{j}^{\prime \prime}$. In order to better underline this interesting effect, we state our answers to Question B for real coefficients. These results hold true for complex coefficients with imaginary parts which satisfy the same hypotheses as in Theorem 1.6 or Proposition 1.8.

Theorem 1.11. Let us consider the problem (1.1) in the case $1<p \leq 3$ under the assumptions (1.4), (1.9) + , (1.3). Let the leading coefficients $a_{2 p}(t), b_{p}(t)$ be Lipschitz continuous functions and let us take real valued $a_{p+j}(t, x), b_{j}(t, x), 1 \leq$ $j \leq p-1$, such that

$$
\begin{aligned}
& a_{p+j} \in C^{0, \alpha_{p+j}}\left([0, T] ; \mathcal{B}^{\infty}\right), b_{j} \in C^{0, \beta_{j}}\left([0, T] ; \mathcal{B}^{\infty}\right), \\
& \left|a_{p+j}\right| \leq C\langle x\rangle^{-\left(j-p \alpha_{p+j}\right) /(p-1)},\left|b_{j}\right| \leq C\langle x\rangle^{-\left(j-p \beta_{j}\right) /(p-1)}, \\
& 0<\alpha_{p+j}, \beta_{j}<j / p, j=1, \ldots, p-1 .
\end{aligned}
$$

For $p>2$, let $\partial_{x} a_{2 p-1}, \partial_{x} b_{p-1}$ be such that

$$
\left|\partial_{x} a_{2 p-1}\right|+\left|\partial_{x} b_{p-1}\right| \leq\langle x\rangle^{-(p-2) /(p-1)} .
$$

Furthermore, still for $p>2$, if $\alpha_{2 p-1}<(p-2) / p$, respectively $\beta_{p-1}<(p-2) / p$, then let us assume

$$
\begin{aligned}
& \left|\partial_{x x} a_{2 p-1}\right| \leq C\langle x\rangle^{-\left(p-2-p \alpha_{2 p-1}\right) /(p-1)}, \\
& \quad \text { respectively }\left|\partial_{x x} b_{p-1}\right| \leq C\langle x\rangle^{-\left(p-2-p \beta_{p-1}\right) /(p-1)},
\end{aligned}
$$

and, if $\alpha_{2 p-1}<(p-2-1 / 2) / p$, respectively $\beta_{p-1}<(p-2-1 / 2) / p$, also

$$
\begin{aligned}
\left|\partial_{x x x} a_{2 p-1}\right| \leq C\langle x\rangle^{-\left(p-2-1 / 2-p \alpha_{2 p-1}\right) /(p-1)} & \\
& \text { respectively }\left|\partial_{x x x} b_{p-1}\right| \leq C\langle x\rangle^{-\left(p-2-1 / 2-p \beta_{p-1}\right) /(p-1)} .
\end{aligned}
$$

Then, the problem (1.1) is well-posed in $H^{s}$.

Remark 1.12. For $\alpha_{p+j}=\beta_{j}=j / p$ in (1.16) (sufficient Hölder regularity, no necessity of decay for $a_{p+j}$ and $b_{j}$ ), the result of Theorem 1.11 reduces to the particular case $a_{p+j}^{\prime \prime}=b_{j}^{\prime \prime}=0$ of real coefficients in Theorem 1.6. For $\alpha_{p+j}=$ $\beta_{j}=0$ (no Hölder continuity, fastest decay) the well-posedness holds true in $H^{\infty}$ with complex $a_{k}, b_{j}$. For merely continuous coefficients, there is not any gain from assuming them to be real valued; cf. the assumptions on the imaginary parts $a_{p+j}^{\prime \prime}, b_{j}^{\prime \prime}$ in Proposition 1.8 


\section{Preliminary Results and First order EQUAtions}

In this section we state some preliminary results and deal with first order (in time) $p$-evolution operators as in (1.5).

We need to introduce pseudo-differential operators $p\left(x, D_{x}\right)$ of order $m$ on $\mathbb{R}$ with symbols $p(x, \xi)$ in the standard class $S^{m}$ defined by

$$
\left|p_{(\beta)}^{(\alpha)}(x, \xi)\right| \leq C_{\alpha, \beta, h}\langle\xi\rangle_{h}^{m-\alpha}, p_{(\beta)}^{(\alpha)}:=\partial_{\xi}^{\alpha} D_{x}^{\beta} p,\langle\xi\rangle_{h}:=\sqrt{h^{2}+\xi^{2}}, h \geq 1 .
$$

These are bounded operators from $H^{s+m}$ to $H^{s}$ for any $s$. In particular, we also use families of symbols $\Lambda(x, \xi)$ such that

$$
|\Lambda(x, \xi)| \leq C+\delta \log \langle\xi\rangle_{h}, \quad\left|\Lambda_{(\beta)}^{(\alpha)}(x, \xi)\right| \leq \delta_{\alpha, \beta}\langle\xi\rangle_{h}^{-\alpha}, \alpha+\beta \geq 1,
$$

with constants $C, \delta$ and $\delta_{\alpha, \beta}$ independent of the family parameter $h$.

Proposition 2.1. Let $\Lambda(x, \xi)$ satisfy (2.2). Then, the operator $e^{\Lambda}$ with symbol $e^{\Lambda(x, \xi)} \in S^{\delta}$ is invertible for a large enough $h$.

Proof. Let us take $e^{-\Lambda}$ with symbol $e^{-\Lambda(x, \xi)}$. We have

$$
e^{\Lambda} e^{-\Lambda}=I-r\left(x, D_{x}\right)
$$

with principal symbol of $r$ given by

$$
r_{-1}(x, \xi)=D_{x} \Lambda(x, \xi) \partial_{\xi} \Lambda(x, \xi) .
$$

We have $\left|r_{(\beta)}^{(\alpha)}(x, \xi)\right| \leq C_{\alpha, \beta}\langle\xi\rangle_{h}^{-1-\alpha}$ with $C_{\alpha, \beta}$ independent on $h$, so we also have

$$
\left|r_{(\beta)}^{(\alpha)}(x, \xi)\right| \leq C_{\alpha, \beta} h^{-1}\langle\xi\rangle_{h}^{-\alpha}
$$

and we can fix a large $h$ in order to have a bounded operator $r\left(x, D_{x}\right)$ on $H^{s}$ with norm $\left\|r\left(x, D_{x}\right)\right\|<1$. From this, $I-r\left(x, D_{x}\right)$ is invertible by Neumann series and its inverse operator

$$
q\left(x, D_{x}\right)=\sum_{j=0}^{\infty} r^{j}\left(x, D_{x}\right)
$$

has symbol in $S^{0}$. The operator $e^{-\Lambda} q$ is the right inverse of $e^{\Lambda}$. By similar arguments one proves the existence of a left inverse, so $e^{-\Lambda} q$ is the (two-sided) inverse operator.

We use also the following result for $k \times k$ matrix operators which shows that an operator of order $m$ with positive Hermitian symbol is a positive operator modulo an error of order $m-1$. The asymptotic expansion of the error term is very important for our applications in the case $p>2$.

Theorem 2.2 ([8], page 134). Let $Q(x, \xi)$ be a $k \times k$ matrix of symbols in $S^{m}$, $k \geq 1$, and assume that its Hermitian part satisfies

$$
\left(Q(x, \xi)+Q^{*}(x, \xi)\right) / 2 \geq 0 .
$$

Then there is a positive $k \times k$ matrix operator $P\left(x, D_{x}\right)$ of order $m$,

$$
2 \Re(P u, u) \geq 0, u \in H^{m},
$$


such that

$$
\begin{aligned}
& Q\left(x, D_{x}\right)=P\left(x, D_{x}\right)+R\left(x, D_{x}\right), \quad R(x, \xi) \in S^{m-1}, \\
& R(x, \xi) \sim \psi_{1}(\xi) D_{x} Q(x, \xi)+\sum_{\alpha+\beta \geq 2} \psi_{\alpha, \beta}(\xi) \partial_{\xi}^{\alpha} D_{x}^{\beta} Q(x, \xi), \\
& \psi_{1} \in S^{-1}, \psi_{\alpha, \beta} \in S^{(\alpha-\beta) / 2} .
\end{aligned}
$$

This implies the well-known sharp Gårding inequality

$$
2 \Re(Q u, u) \geq-C\|u\|_{(m-1) / 2}^{2}
$$

for a matrix operator satisfying (2.4). We deal with matrices in the next section. For scalar operators with positive symbol, the stronger Fefferman-Phong inequality

$$
2 \Re(Q u, u) \geq-C\|u\|_{(m-2) / 2}^{2}
$$

holds true.

Let us now consider the Cauchy problem for an operator $L_{1}$ as in (1.5), that is,

$$
\left\{\begin{array}{l}
D_{t} u+e_{p}(t) D_{x}^{p} u+\sum_{j=0}^{p-1} e_{j}(t, x) D_{x}^{j} u=0 \\
u(0, x)=u_{0} .
\end{array}\right.
$$

We say that the problem is well-posed in $H^{s}$ if for any $u_{0} \in H^{s}$ there is a unique solution $u \in C\left([0, T] ; H^{s}\right)$. If there is a unique solution $u \in C\left([0, T] ; H^{s-\delta}\right), \delta>0$, then we say that (2.6) is well-posed in $H^{\infty}$ (with a loss of derivatives).

We assume that the leading coefficient is real and such that

$$
e_{p} \in C([0, T] ; \mathbb{R}), e_{p}(t) \geq 0 .
$$

The lower order coefficients are complex valued and such that

$$
e_{j} \in C\left([0, T] ; \mathcal{B}^{\infty}\right), \quad j=0, \ldots, p-1 .
$$

Theorem 2.3. Let us consider the problem (2.6) with $1<p \leq 3$ under the assumptions (2.7) and (2.8). Let us write $e_{j}=e_{j}^{\prime}+i e_{j}^{\prime \prime}$ with real $e_{j}^{\prime}, e_{j}^{\prime \prime}$. For the imaginary part $e_{j}^{\prime \prime}$, let us assume

$$
\left|e_{p-1}^{\prime \prime}\right| \leq C e_{p}(t)\langle x\rangle^{-\sigma}, \sigma \geq 1
$$

and, for $p>2$,

$$
\left|\partial_{x} e_{p-1}^{\prime}\right|+\left|e_{p-2}^{\prime \prime}\right| \leq C e_{p}(t)\langle x\rangle^{-(p-2) /(p-1)} .
$$

Then the Cauchy problem (2.6) is well-posed in Sobolev spaces. In particular, if (2.9) is fulfilled with $\sigma>1$, then the problem (2.6) is well-posed in $H^{s}$. If (2.9) is fulfilled with $\sigma=1$, then the problem (2.6) is well-posed in $H^{\infty}$ with a loss of derivatives.

Remark 2.4. If the leading coefficient $e_{p}(t)$ never vanishes in $[0, T]$, then (2.9) and (2.10) reduce to

$$
\left|e_{p-1}^{\prime \prime}\right| \leq C^{\prime}\langle x\rangle^{-\sigma},\left|\partial_{x} e_{p-1}^{\prime}\right|+\left|e_{p-2}^{\prime \prime}\right| \leq C^{\prime}\langle x\rangle^{-(p-2) /(p-1)} .
$$


Proof of Theorem 2.3. Our aim is to obtain the well-posedness in $H^{s}$ or $H^{\infty}$ of problem (2.6) for the operator $L_{1}$ as in (1.5), by proving the well-posedness in $H^{s}$ of the Cauchy problem for a transformed operator

$$
L_{1}^{\Lambda}:=\left(e^{\Lambda}\right)^{-1} L_{1} e^{\Lambda},
$$

where $\Lambda$ is real valued and satisfies (2.2). The symbols of $e^{\Lambda},\left(e^{\Lambda}\right)^{-1}$ are in $S^{\delta}$ and this brings a loss of derivatives for $\delta>0$. In the case $\sigma>1$ in (2.9), we can take $\delta=0$ in the change of variable, so that the well-posedness in $H^{s}$ holds for (2.6).

Let us write $i L_{1}=\partial_{t}+i K\left(t, x, D_{x}\right)$, that is,

$$
K\left(t, x, D_{x}\right)=e_{p}(t) D_{x}^{p}+\sum_{j=0}^{p-1} e_{j}(t, x) D_{x}^{j}
$$

Since

$$
i L_{1}^{\Lambda}=\partial_{t}+i K^{\Lambda}, \quad K^{\Lambda}=\left(e^{\Lambda}\right)^{-1} K e^{\Lambda},
$$

we seek $\Lambda(x, \xi)$ such that $i K^{\Lambda}$ is bounded from below in $L^{2}$ uniformly for $t \in[0, T]$ :

$$
2 \Re\left(i K^{\Lambda} u, u\right) \geq-C\|u\|^{2}, u \in H^{p} .
$$

From this, the energy method gives the well-posedness in $L^{2}$ of the Cauchy problem for $L_{1}^{\Lambda}$. Provided that $\left\langle D_{x}\right\rangle^{s} i K^{\Lambda}\left\langle D_{x}\right\rangle^{-s}$ also satisfies (2.13), the well-posedness in $H^{s}$ follows.

Let us write $i K$ as the sum

$$
i K=H_{K}+A_{K}, \quad H_{K}=\left(i K+(i K)^{*}\right) / 2, A_{K}=\left(i K-(i K)^{*}\right) / 2
$$

of its Hermitian and anti-Hermitian parts. In order to obtain (2.13), we consider two zones in the phase space: the support of $\varrho\left(\langle x\rangle /\langle\xi\rangle_{h}^{p-1}\right)$ and the support of $\left(1-\varrho\left(\langle x\rangle /\langle\xi\rangle_{h}^{p-1}\right)\right)$ with $\varrho \in C_{0}^{\infty}$ a cutoff function, $0 \leq \varrho(y) \leq 1, \varrho(y)=1$ in a neighborhood of $y=0$. Conditions (2.9) and (2.10) imply

$$
\left(1-\varrho\left(\langle x\rangle /\langle\xi\rangle_{h}^{p-1}\right)\right) H_{K}(t, x, \xi) \in C\left([0, T] ; S^{0}\right) ;
$$

hence, we have to transform only $\varrho H_{K}$ into a bounded from below operator. We define the symbol $\Lambda$ as a sum of $p-1$ terms $\Lambda_{p-j}, j=1, \ldots, p-1$, each one related to the part of positive order $j-h$ in the expansion of the Hermitian part $H_{K}$. The first term is

$$
\Lambda_{p-1}=M_{p-1} \omega(\xi / h) \int_{0}^{x}\langle y\rangle^{-\sigma} \varrho\left(\langle y\rangle /\langle\xi\rangle_{h}^{p-1}\right) d y
$$

with $M_{p-1}$ a large constant and $\omega(y)$ a smooth function with $\omega(y)=0$ for $|y| \leq 1$, $\omega(y)=|y|^{p-1} / y^{p-1}$ for $|y| \geq 2$. Such a symbol $\Lambda_{p-1}$ satisfies (2.2) for $\sigma=1$ whereas for $\sigma>1$ it belongs to $S^{0}$, still with $\sup _{x, \xi}\left|\Lambda_{(\beta)}^{(\alpha)}(x, \xi)\right|\langle\xi\rangle_{h}^{\alpha}$ independent of $h$. Also taking

$$
\langle x\rangle^{-1} \partial_{\xi} \Lambda_{p-1} \in S^{-p},\langle x\rangle^{\sigma} \partial_{x} \Lambda_{p-1} \in S^{0}
$$

and (2.3) into account, the Hermitian part $H_{K^{\Lambda_{p-1}}}$ of $i K^{\Lambda_{p-1}}$ is given by

$$
\begin{aligned}
& H_{K^{\Lambda_{p-1}}}(t, x, \xi) \\
& \quad=\varrho\left(\langle x\rangle /\langle\xi\rangle_{h}^{p-1}\right)\left[p M_{p-1} e_{p}(t)|\xi|^{p-1}\langle x\rangle^{-\sigma}-e_{p-1}^{\prime \prime}(t, x) \xi^{p-1}\right]+Q_{p-2}(t, x, \xi),
\end{aligned}
$$


with $Q_{p-2}(t, x, \xi) \in C\left([0, T] ; S^{p-2}\right)$. Taking a sufficiently large $M_{p-1}$, in view of (2.9) and thanks to Theorem 2.2. there are a positive operator $P_{p-1}\left(t, x, D_{x}\right)$ of order $p-1$ and a remainder $R_{p-2}\left(t, x, D_{x}\right)$ of order $p-2$ such that

$$
H_{K^{\Lambda}-1}=P_{p-1}+R_{p-2}
$$

hence, considering also the anti-Hermitian part $i e_{p}(t) D_{x}^{p}+A_{p-1}$, we get

$$
\begin{aligned}
i K^{\Lambda_{p-1}}= & i e_{p}(t) D_{x}^{p}+A_{p-1}+P_{p-1}+R_{p-2}, \\
& 2 \Re\left(A_{p-1} u, u\right)=0,2 \Re\left(P_{p-1} u, u\right) \geq 0, R_{p-2} \in C\left([0, T] ; S^{p-2}\right) .
\end{aligned}
$$

In the case $p=2$, this is already sufficient to get (2.13) with $\Lambda=\Lambda_{p-1}$.

If $p-2>0$, then we have to add a term $\Lambda_{p-2}$ in the expansion of $\Lambda$ and to specify the principal part of the remainder $R_{p-2}$. Since here we consider only $p \leq 3$ and we are dealing with scalar operators, it is important to observe that, taking directly

$$
P_{p-1}^{\prime}(t, x, \xi)=\varrho\left(\langle x\rangle /\langle\xi\rangle_{h}^{p-1}\right)\left[p M_{p-1} e_{p}(t)|\xi|^{p-1}\langle x\rangle^{-\sigma}-e_{p-1}^{\prime \prime}(t, x) \xi^{p-1}\right]
$$

the Fefferman-Phong inequality also gives

$$
\begin{aligned}
& i K^{\Lambda_{p-1}}=i e_{p}(t) D_{x}^{p}+A_{p-1}+P_{p-1}^{\prime}+R_{p-2}^{\prime}, \\
& 2 \Re\left(P_{p-1}^{\prime} u, u\right) \geq-C\|u\|_{(p-3) / 2}^{2}, R_{p-2}^{\prime} \in C\left([0, T] ; S^{p-2}\right),
\end{aligned}
$$

where the principal part of $R_{p-2}^{\prime}$ now does not contain the real term $\psi_{0,2}(\xi) \partial_{x x} e_{p-1}^{\prime \prime} \xi^{p-1}$ which comes in $R_{p-2}$ of (2.15) from (2.5). This allows us to not include $\partial_{x x} e_{p-1}^{\prime \prime}$ in (2.10) for the present case $p \leq 3$. We define $\Lambda_{p-2}$ by

$$
\Lambda_{p-2}(x, \xi)=M_{p-2}\langle\xi\rangle_{h}^{-1} \omega(\xi / h) \int_{0}^{x}\langle y\rangle^{-(p-2) /(p-1)} \varrho\left(\langle y\rangle /\langle\xi\rangle_{h}^{p-1}\right) d y,
$$

which belongs to $S^{0}$ with semi-norms independent of $h$ and compute the expansion of the Hermitian part $H_{K^{\Lambda}}, \Lambda=\Lambda_{p-1}+\Lambda_{p-2}$. Also taking

$$
\langle x\rangle^{(p-2) /(p-1)} \partial_{x} \Lambda_{p-2},\langle x\rangle^{-1 /(p-1)} \Lambda_{p-2} \in S^{-1}
$$

and (2.3) into account, we have

$$
\begin{aligned}
H_{K^{\Lambda}}(t, x, \xi)=P_{p-1}^{\prime}(t, x, \xi)+\varrho\left(\langle x\rangle /\langle\xi\rangle_{h}^{p-1}\right) Q_{p-2}^{\prime}(t, x, \xi) & \\
& +i(p-1) \partial_{x} e_{p-1}^{\prime \prime}(t) \xi^{p-2} / 2+R_{p-3}(t, x, \xi)
\end{aligned}
$$

with $P_{p-1}^{\prime}$ as in (2.16),

$$
\begin{aligned}
& Q_{p-2}^{\prime}(t, x, \xi)=p M_{p-2} e_{p}(t)|\xi|^{p-1}\langle\xi\rangle_{h}^{-1}\langle x\rangle^{-(p-2) /(p-1)} \\
& \quad+\xi^{p-2}\left[-e_{p-2}^{\prime \prime}(t)-(p-1) \partial_{x} e_{p-1}^{\prime}(t) / 2+(p-1) M_{p-1} e_{p-1}^{\prime}(t)\langle x\rangle^{-\sigma}\right],
\end{aligned}
$$

and

$$
R_{p-3} \in C\left([0, T] ; S^{p-3}\right) .
$$

From (2.10), we can choose the constant $M_{p-2}$ in order to have

$$
Q_{p-2}^{\prime}(t, x, \xi) \geq 0 .
$$


Hence, denoting by $i e_{p}(t) D_{x}^{p}+A_{p-1}^{\prime}$ the anti-Hermitian part of $i K^{\Lambda}$ and by $A_{p-2}$ the anti-Hermitian part of $i(p-1) \partial_{x} e_{p-1}^{\prime \prime}(t) D_{x}^{p-2} / 2$, there is a positive operator $P_{p-2}$ of order $p-2$ such that

$$
\begin{aligned}
& i K^{\Lambda}=i e_{p}(t) D_{x}^{p}+A_{p-1}^{\prime}+P_{p-1}^{\prime}+A_{p-2}+P_{p-2}+R_{p-3}^{\prime}, \\
& 2 \Re\left(i e_{p}(t) D_{x}^{p} u+A_{p-1}^{\prime} u+A_{p-2} u, u\right)=0, \\
& 2 \Re\left(P_{p-1}^{\prime} u, u\right) \geq-C\|u\|_{(p-3) / 2}^{2}, 2 \Re\left(P_{p-2} u, u\right) \geq 0,
\end{aligned}
$$

and $R_{p-3}^{\prime} \in C\left([0, T] ; S^{p-3}\right)$. The expansion (2.19) gives inequality (2.13) also for $p=3$.

In order to complete the proof for any $H^{s}$, we observe that it is sufficient to choose constants $M_{p-1}=M_{p-1}(s), M_{p-2}=M_{p-2}(s)$ in order to have the same bound (2.13) for $\left\langle D_{x}\right\rangle^{s} i K^{\Lambda}\left\langle D_{x}\right\rangle^{-s}$.

\section{Proofs of the MAin Results}

In this section we consider an operator $L$ as in (1.2) and we prove Theorem 1.6, Proposition 1.8, Theorem 1.10 and Theorem 1.11 .

We reduce the equation $L u=0$ to a first order (in $\partial_{t}$ ) $2 \times 2$ system. In doing so, we need a partial factorization of $L$. We describe in detail the procedure under the assumptions of Theorem [1.6. then, in the proofs of the other results, we sketch the necessary changes.

Lemma 3.1. Let us consider the operator $L$ given by (1.2) under the assumptions of Theorem 1.6 and let

$$
e_{p}^{ \pm}(t)=b_{p}(t) \pm \sqrt{b_{p}^{2}(t)-a_{2 p}(t)}
$$

such that $-e_{p}^{+}(t) \xi^{p}$ and $-e_{p}^{-}(t) \xi^{p}$ are the real distinct roots of the principal symbol of $L$. Then

$$
L=L_{1}^{-}\left(t, x, D_{t}, D_{x}\right) L_{1}^{+}\left(t, x, D_{t}, D_{x}\right)+R_{p}\left(t, x, D_{x}\right)+R_{0}\left(t, x, D_{x}\right) D_{t},
$$

where

$$
L_{1}^{ \pm}=D_{t}+e_{p}^{ \pm}(t) D_{x}^{p}+\sum_{j=1}^{p-1} e_{j}^{ \pm}\left(t, x, D_{x}\right) D_{x}^{j}
$$

with $e_{j}^{ \pm} \in C\left([0, T] ; S^{0}\right)$ such that

$$
\left|\Im e_{p-1}^{ \pm}\right| \leq C\langle x\rangle^{-\sigma}
$$

and, for $p>2$, such that

$$
\left|\partial_{x} \Re e_{p-1}^{ \pm}\right|+\left|\Im e_{p-2}^{ \pm}\right| \leq C\langle x\rangle^{-(p-2) /(p-1)},
$$

and where

$$
R_{p} \in L^{\infty}\left([0, T] ; S^{p}\right), R_{0} \in L^{\infty}\left([0, T] ; S^{0}\right) .
$$

Proof. The first step in the proof is to factorize the principal part of $L$ obtaining

$$
L=\left(D_{t}+e_{p}^{-}(t) D_{x}^{p}\right)\left(D_{t}+e_{p}^{+}(t) D_{x}^{p}\right)+R_{2 p-1}\left(t, x, D_{x}\right)+R_{p-1}\left(t, x, D_{x}\right) D_{t},
$$

where the principal part of the remainder is

$$
a_{2 p-1}(t, x) D_{x}^{2 p-1}+b_{p-1}(t, x) D_{x}^{p-1} D_{t} .
$$


If also $a_{2 p-1}$ and $b_{p-1}$ were Lipschitz continuous in $t$, then one could perform directly a second step of factorization. Since these coefficients are only $C^{0,(p-1) / p}$, we introduce the regularization

$$
\tilde{a}_{2 p-1}(t, x, \xi)=\int_{-\infty}^{+\infty} a_{2 p-1}(\tau, x) g\left((t-\tau)\langle\xi\rangle_{h}^{p}\right)\langle\xi\rangle_{h}^{p} d \tau
$$

where $g(y)$ is a cutoff function, $0 \leq g(y) \leq 1, \int_{-\infty}^{+\infty} g(y) d y=1$, and $a_{2 p-1}(\tau, x)=$ $a_{2 p-1}(0, x)$ for $\tau \leq 0, a_{2 p-1}(\tau, x)=a_{2 p-1}(T, x)$ for $\tau \geq T$. We also define $\tilde{b}_{p-1}$ in the same way. From the Hölder continuity in $t$ of $a_{2 p-1}(t, x)$ and $b_{p-1}(t, x)$, we have

$$
\begin{aligned}
\tilde{a}_{2 p-1}, \tilde{b}_{p-1} \in C\left([0, T] ; S^{0}\right) \cap & C^{1}\left([0, T] ; S^{1}\right), \\
& \tilde{a}_{2 p-1}-a_{2 p-1}, \tilde{b}_{p-1}-b_{p-1} \in C\left([0, T] ; S^{-p+1}\right) .
\end{aligned}
$$

Modulo a term of order zero, the principal symbol of $R_{2 p-1}+R_{p-1} D_{t}$ can be represented by

$$
\tilde{a}_{2 p-1}(t, x, \xi) \xi^{2 p-1}+\tilde{b}_{p-1}(t, x, \xi) \xi^{p-1} \tau
$$

which is differentiable in $t$. Taking the solution $e_{p-1}^{ \pm}(t, x, \xi)$ of the linear system

$$
\left\{\begin{array}{l}
e_{p}^{-} e_{p-1}^{+}+e_{p}^{+} e_{p-1}^{-}=\tilde{a}_{2 p-1} \\
e_{p-1}^{+}+e_{p-1}^{-}=\tilde{b}_{p-1}
\end{array}\right.
$$

we have

$$
L=\left(D_{t}+e_{p}^{-} D_{x}^{p}+e_{p-1}^{-} D_{x}^{p-1}\right)\left(D_{t}+e_{p}^{+} D_{x}^{p}+e_{p-1}^{+} D_{x}^{p-1}\right)+R_{2 p-2}+R_{p-2} D_{t}
$$

which gives (3.2) together with (3.4) for $p=2$, taking (1.11) into account.

For $p>2$, we take the symbols $\tilde{a}_{2 p-2}$ and $\tilde{b}_{p-2}$, which are such that

$$
\begin{aligned}
\tilde{a}_{2 p-2}, \tilde{b}_{p-2} \in C^{1}\left([0, T] ; S^{0}\right) & \cap C^{1}\left([0, T] ; S^{2}\right), \\
& \tilde{a}_{2 p-2}-a_{2 p-2}, \tilde{b}_{p-2}-b_{p-2} \in C\left([0, T] ; S^{-p+2}\right),
\end{aligned}
$$

and represent the principal symbols of $R_{2 p-2}+R_{p-2} D_{t}$ by

$$
r_{2 p-2}^{0} \xi^{2 p-2}+\tilde{b}_{p-2} \xi^{p-2} \tau, \quad r_{2 p-2}^{0}=\tilde{a}_{2 p-2}+e_{p-1}^{-} e_{p-1}^{+}+p e_{p}^{-} D_{x} e_{p-1}^{+} .
$$

Since we have $\left|\partial_{x} \Re e_{p-1}^{ \pm}\right| \leq C\langle x\rangle^{-(p-2) /(p-1)}$ from (1.12), it follows that the imaginary part of $r_{2 p-2}^{0}$ also satisfies

$$
\left|\Im r_{2 p-2}^{0}\right| \leq C\langle x\rangle^{-(p-2) /(p-1)}
$$

as $\Im \tilde{b}_{p-2}$ does, still from (1.12). Now taking the solution $e_{p-2}^{ \pm}(t, x, \xi)$ of the linear system

$$
\left\{\begin{array}{l}
e_{p}^{-} e_{p-2}^{+}+e_{p}^{+} e_{p-2}^{-}=r_{2 p-2}^{0} \\
e_{p-2}^{+}+e_{p-2}^{-}=\tilde{b}_{p-2}
\end{array}\right.
$$

we have

$$
\begin{array}{r}
L=\left(D_{t}+e_{p}^{-} D_{x}^{p}+e_{p-1}^{-} D_{x}^{p-1}+e_{p-2}^{-} D_{x}^{p-2}\right)\left(D_{t}+e_{p}^{+} D_{x}^{p}+e_{p-1}^{+} D_{x}^{p-1+} e_{p-2}^{+} D_{x}^{p-2}\right) \\
+R_{2 p-3}+R_{p-3} D_{t},
\end{array}
$$

which gives (3.2) together with (3.4) and (3.5) for $p=3$. Since we are considering $1<p \leq 3$, we can stop the factorization procedure. 
Lemma 3.2. Let us consider the operator $L$ given by (1.2) under the assumptions of Theorem 1.6 and let us denote by

$$
L_{1}^{ \pm}=D_{t}+K_{1}^{ \pm}\left(t, x, D_{x}\right)
$$

the operators in (3.3), that is,

$$
K_{1}^{ \pm}\left(t, x, D_{x}\right)=e_{p}^{ \pm}(t) D_{x}^{p}+\sum_{j=1}^{p-1} e_{j}^{ \pm}\left(t, x, D_{x}\right) D_{x}^{j} .
$$

Then, the scalar equation $L u=0$ is equivalent to the $2 \times 2$ system $S U=0$,

$$
S=D_{t}+K\left(t, x, D_{x}\right)+E_{0}\left(t, x, D_{x}\right),
$$

with

$$
K=\left(\begin{array}{cc}
K^{+} & 0 \\
0 & K^{-}
\end{array}\right), \quad K^{+}=K_{1}^{+}+\left[\left\langle D_{x}\right\rangle^{p}, K_{1}^{+}\right]\left\langle D_{x}\right\rangle^{-p}, K^{-}=K_{1}^{-},
$$

$\left[\left\langle D_{x}\right\rangle^{p}, K_{1}^{+}\right]$the commutator of $\left\langle D_{x}\right\rangle^{p}$ and $K_{1}^{+}$, and with

$$
E_{0}(t, x, \xi)=\left(\begin{array}{ll}
e_{11}^{0}(t, x, \xi) & e_{12}^{0}(t, x, \xi) \\
e_{21}^{0}(t, x, \xi) & e_{22}^{0}(t, x, \xi)
\end{array}\right), e_{j k}^{0}(t, x, \xi) \in L^{\infty}\left([0, T] ; S^{0}\right) .
$$

Remark 3.3. The term $\left[\left\langle D_{x}\right\rangle^{p}, K_{1}^{+}\right]\left\langle D_{x}\right\rangle^{-p}$ in $K^{+}$is of order $p-2$ since $e_{p}^{+}$does not depend on $x$. For $p=2$, one can move it from the diagonal part $D_{t}+K$ of the system $S$ into the matrix $E_{0}$. For $p>2$, we observe that, from (1.12) and (3.9), its principal imaginary part $p\left(1+\xi^{2}\right)^{-1} \xi^{p} \partial_{x} \Re e_{p-1}^{+}$is also bounded by $C\langle x\rangle^{-(p-2) /(p-1)}\langle\xi\rangle^{p-2}$ as $\Im e_{p-2}^{ \pm} \xi^{p-2}$.

Proof of Lemma 3.2. For a scalar unknown $u$ we define the vector $U={ }^{t}\left(u_{0}, u_{1}\right)$ by

$$
u_{0}=\left\langle D_{x}\right\rangle^{p} u, u_{1}=L_{1}^{+} u
$$

so that, from (3.2), the scalar equation $L u=0$ is equivalent to the system $S_{1} U=0$ with

$$
S_{1}=D_{t}+\left(\begin{array}{cc}
K^{+} & -\left\langle D_{x}\right\rangle^{p} \\
0 & K^{-}
\end{array}\right)+\left(\begin{array}{cc}
0 & 0 \\
\left(R_{p}-R_{0} K_{1}^{+}\right)\left\langle D_{x}\right\rangle^{-p} & R_{0}
\end{array}\right) .
$$

Now we perform $p-1$ steps of diagonalization. In view of (1.4), the matrix

$$
\left(\begin{array}{cc}
K^{+} & -\langle\xi\rangle^{p} \\
0 & K^{-}
\end{array}\right)
$$

can be smoothly diagonalized for large $|\xi|$, say $|\xi| \geq R$, by

$$
N_{0}(t, x, \xi)=\left(\begin{array}{cc}
1 & n_{0}(t, x, \xi) \\
0 & 1
\end{array}\right), n_{0}(t, x, \xi)=\langle\xi\rangle^{p} /\left(K^{+}-K^{-}\right),
$$

which is in $C^{1}\left([0, T] ; S^{0}\right)$ by (3.8) and (3.10). For the system $S_{1}$ in (3.16) we have

$$
N_{0}^{-1} S_{1} N_{0}=S
$$

with $S$ as in (3.13) modulo a term

$$
\left(\begin{array}{cc}
0 & z_{p-2} \\
0 & 0
\end{array}\right)
$$

where the principal part

$$
z_{p-2}^{0}=\partial_{\xi} K^{+} D_{x} n_{0}-\partial_{\xi} n_{0} D_{x} K^{-}
$$


of $z_{p-2}$ is of order $p-2$, since $e_{p}^{ \pm}$does not depend on $x$. For $p=2$, this first step of diagonalization is sufficient. For $p>2$, we perform a second step by means of the operator with symbol

$$
N_{1}=\left(\begin{array}{cc}
1 & -z_{p-2}^{0} /\left(K^{+}-K^{-}\right) \\
0 & 1
\end{array}\right),|\xi| \geq R,
$$

obtaining an error of order $p-3$. Since we are considering $1<p \leq 3$, we can stop the diagonalization procedure.

Proof of Theorem 1.6. We prove the well-posedness of the Cauchy problem (1.1) for the scalar operator $L$ by proving the well-posedness of the equivalent first order (in time) problem

$$
\left\{\begin{array}{l}
S U(t, x)=0 \\
U(0, x)=U_{0}(x)
\end{array}\right.
$$

for the system $S$ in (3.13). In doing so, we can apply Theorem 2.3 (in its pseudodifferential form) to the diagonal part $D_{t}+K$ of $S=D_{t}+K+E_{0}$ thanks to (3.4), (3.5) and, for $p>2$, also taking Remark 3.3 into account.

From (1.9), the functions $e_{p}^{ \pm}(t)$ do not vanish. Let

$$
\delta_{ \pm}=e_{p}^{ \pm}(t) /\left|e_{p}^{ \pm}(t)\right|
$$

be the respective signs and, for $\Lambda$ as in (2.11) constructed in the proof of Theorem 2.3. let us consider the transformed system

$$
S^{\Lambda}:=\left(\begin{array}{cc}
e^{\delta_{+} \Lambda} & 0 \\
0 & e^{\delta_{-} \Lambda}
\end{array}\right)^{-1} S\left(\begin{array}{cc}
e^{\delta_{+} \Lambda} & 0 \\
0 & e^{\delta_{-} \Lambda}
\end{array}\right)
$$

We have

$$
S^{\Lambda}=D_{t}+K^{\Lambda}+E_{0}^{\Lambda}
$$

where

$$
2 \Re\left(i K^{\Lambda} U, U\right) \geq-C\|U\|^{2}, U \in H^{p} .
$$

The energy estimate gives the well-posedness in $H^{s}$ of the Cauchy problem for $S^{\Lambda}$, which corresponds to the well-posedness of (3.19) in $H^{s}$ for $\sigma>1$ in (1.11) and in $H^{\infty}$ for $\sigma=1$, provided that $E_{0}^{\Lambda}$ is of order zero. This is true, independently of the signs $\delta_{ \pm}$, for $\sigma>1$ because $\Lambda$ is of order 0 in this case. For $\sigma=1$, here we need to specify (1.9) into (1.9) + in order to avoid terms of positive order in the anti-diagonal part of $E_{0}^{\Lambda}$. In fact, for $\delta_{+}=\delta_{-}$, the change of variable in (3.21) is of scalar type and we have $E_{0}^{\Lambda}=E_{0}+E_{-1}$ with $E_{-1}$ a matrix operator of order -1 .

Proof of Proposition 1.8. Here the assumption (1.10) is weakened into (1.10) . In Lemma 3.1, this allows us to include only the real parts of $a_{p+j} D_{x}^{p+j}, b_{j} D_{x}^{j} D_{t}$, $1 \leq j \leq p-1$, in the factorization of $L$ and to leave the imaginary parts in the remainder. Consequently, in Lemma 3.2, the system $S$ is now given by

$$
S=D_{t}+K\left(t, x, D_{x}\right)+\sum_{j=0}^{p-1} F_{j}\left(t, x, D_{x}\right),
$$


where:

- the operators $K_{1}^{ \pm}$in the diagonal matrix $K$ defined by (3.14) have real full symbols; in particular, the Hermitian part $\left(i K+(i K)^{*}\right) / 2$ is of order $p-2$ and, from (1.12), the principal part $H_{K}^{0}$ is such that

$$
\left|H_{K}^{0}\right| \leq C\langle x\rangle^{-(p-2) /(p-1)}\langle\xi\rangle^{p-2}
$$

for $p>2$;

- the full matrices $F_{j}, 0 \leq j \leq p-1$, are of order $j$ with

$$
\left|F_{p-1}\right| \leq C\langle x\rangle^{-\sigma}\langle\xi\rangle^{p-1}
$$

from (1.11) and with

$$
\begin{gathered}
\langle\xi\rangle^{-1}\left|\partial_{x} F_{p-1}\right|+\langle\xi\rangle^{-1}\left|\partial_{x x} F_{p-1}\right|+\left|F_{p-2}\right| \leq C\langle x\rangle^{-(p-2) /(p-1)}\langle\xi\rangle^{p-2} \\
\left|\partial_{x x x} F_{p-1}\right| \leq C\langle x\rangle^{-(p-2-1 / 2) /(p-1)}\langle\xi\rangle^{p-1}
\end{gathered}
$$

from (1.12), (1.12) ${ }^{\prime}$ and (1.13) for $p>2$.

In view of (1.9) $)_{+}$, we have $\delta_{+}=\delta_{-}$in (3.20), say $\delta_{+}=\delta_{-}=+1$ : if $\delta_{+}=\delta_{-}=-1$, then one just changes $\Lambda$ into $-\Lambda$ in what follows. This means that the change of variable in (3.21) is given by the scalar operator $e^{\Lambda}$ and for a full matrix operator $F=F\left(x, D_{x}\right)$ we have the same expansion

$$
F^{\Lambda}=F+\text { lower order terms }
$$

of $F^{\Lambda}=\left(e^{\Lambda}\right)^{-1} F e^{\Lambda}$ as we have for a scalar $F$. From this, we can follow the same strategy as in the proof of Theorem 2.3 since the sharp Gårding inequality holds true for the system. We cannot use the Fefferman-Phong inequality here, so the expansion of the error term in (2.5) leads us to assume (1.12) ${ }^{\prime}$ and (1.13) besides (1.12) in order to provide (3.25) and (3.26) for $p>2$.

Let us take $\Lambda_{p-1}$ as in (2.14) with a sufficiently large constant $M_{p-1}$. From (3.24), Theorem 2.2 and taking the order $p-2$ of $\left(i K+(i K)^{*}\right) / 2$ into account, there are an anti-Hermitian matrix operator $A_{p}$ of order $p$, a positive matrix operator $P_{p-1}$ of order $p-1$ and a matrix operator $Q_{p-2}$ of order $p-2$ such that

$$
\left(e^{\Lambda_{p-1}}\right)^{-1} i S e^{\Lambda_{p-1}}=\partial_{t}+A_{p}+P_{p-1}+Q_{p-2}
$$

for the system $S$ in (3.22). For $p=2$, this is already sufficient to obtain the desired well-posedness of (3.19), hence of (1.1), according to the value of $\sigma$ in (1.11). For $p>2$, (3.23) and (2.5), together with (3.25) and (3.26), give

$$
Q_{p-2}=G_{p-2}+G_{p-2-1 / 2}+G_{p-3},
$$

where each $G_{q}$ is of order $q$ and with symbol such that

$$
\left|G_{q}\right| \leq C\langle x\rangle^{-q /(p-1)}\langle\xi\rangle^{q}, q>p-3 .
$$

Following this expansion of $Q_{p-2}$, as in the proof of Theorem 2.3 we add terms to $\Lambda_{p-1}$ by defining

$$
\Lambda=\Lambda_{p-1}+\Lambda_{p-2}+\Lambda_{p-2-1 / 2}
$$

with $\Lambda_{q}$ for $q>p-1$ defined as in (2.18) by

$$
\Lambda_{q}(x, \xi)=M_{q}\langle\xi\rangle_{h}^{q-p+1} \omega(\xi / h) \int_{0}^{x}\langle y\rangle^{-q /(p-1)} \varrho\left(\langle y\rangle /\langle\xi\rangle_{h}^{p-1}\right) d y .
$$


Since the sum of anti-Hermitian operators is anti-Hermitian and the sum of positive operators is positive, we obtain

$$
\left(e^{\Lambda}\right)^{-1} i S e^{\Lambda}=\partial_{t}+A+P+Q_{p-3}
$$

with $A$ anti-Hermitian of order $p, P$ positive of order $p-1$ and $Q_{p-3}$ of order $p-3$. This concludes the proof for $p \leq 3$.

Proof of Theorem 1.10. Under the assumptions of Theorem 1.10, the differentiability of the coefficients with respect to the time variable in (1.15) allows us to perform $N+p$ steps of factorization in Lemma 3.1. instead of $p$ steps, and without any need for the regularizations $\tilde{a}_{k}, \tilde{b}_{j}$ defined in (3.7). This leads to

$$
L=L_{1}^{-}\left(t, x, D_{t}, D_{x}\right) L_{1}^{+}\left(t, x, D_{t}, D_{x}\right)+R_{p-N}\left(t, x, D_{x}\right)+R_{-N}\left(t, x, D_{x}\right) D_{t},
$$

where now, still denoting by $D_{x}^{j}$ an operator with symbol $\xi^{j}$ outside a neighborhood of $\xi=0$ also for $j<0$,

$$
L_{1}^{ \pm}=D_{t}+e_{p}^{ \pm}(t) D_{x}^{p}+\sum_{j=-N+1}^{p-1} e_{j}^{ \pm}\left(t, x, D_{x}\right) D_{x}^{j}
$$

with $e_{j}^{ \pm} \in C\left([0, T] ; S^{0}\right)$ and where the remainders are now such that

$$
R_{p-N} \in C\left([0, T] ; S^{p-N}\right), R_{-N} \in C\left([0, T] ; S^{-N}\right) .
$$

In particular, here $e_{p-1}^{ \pm}(t, x, \xi)$ is the solution of the linear system

$$
\left\{\begin{array}{l}
e_{p}^{-} e_{p-1}^{+}+e_{p}^{+} e_{p-1}^{-}=a_{2 p-1} \\
e_{p-1}^{+}+e_{p-1}^{-}=b_{p-1}
\end{array}\right.
$$

with roots $e_{p}^{ \pm}$in (3.1) of different sign, $e_{p}^{+}>0, e_{p}^{-}<0$, from (1.9) - .

In Lemma 3.2, we still define $K$ as in (3.14), now with

$$
K_{1}^{ \pm}\left(t, x, D_{x}\right)=e_{p}^{ \pm}(t) D_{x}^{p}+\sum_{j=-N+1}^{p-1} e_{j}^{ \pm}\left(t, x, D_{x}\right) D_{x}^{j},
$$

but, after $N+p-1$ steps of diagonalization, we now obtain a system

$$
S=D_{t}+K\left(t, x, D_{x}\right)+E_{-N}\left(t, x, D_{x}\right)
$$

with a non-diagonal part $E_{-N}$ of negative order $-N$. We still apply Theorem 2.3 to the diagonal part $D_{t}+K$ of $S=D_{t}+K+E_{-N}$ as in the proof of Theorem 1.6. now with $\delta_{+}=+1, \delta_{-}=-1$ in (3.21).

Provided that $E_{-N}^{\Lambda}$ in (3.21) is of order less than or equal to zero, the energy method gives the well-posedness in $H^{s}$ of the Cauchy problem for $S^{\Lambda}$, which here corresponds to the well-posedness of (3.19) in $H^{\infty}$ with a loss of derivatives, since now $\sigma=1$ in (1.11). From the different signs of the roots $e_{p}^{ \pm}$and the assumption $\sigma=1$, the order of $E_{-N}^{\Lambda}$ and the loss are given by $-N+2 M_{p-1}$ and $2 M_{p-1}$, respectively, with $M_{p-1}$ the constant in (2.14). From the proof of Theorem 2.3 and the expression of $e_{p-1}^{ \pm}, M_{p-1}$ has to be such that

$$
p\left|e_{p}^{ \pm}\right| M_{p-1} \geq C_{0}\left(1+\left|e_{p}^{ \pm}\right|\right) /\left(e_{p}^{+}-e_{p}^{-}\right),
$$

$C_{0}$ the constant in (1.11), and this is satisfied by taking

$$
2 M_{p-1}=N_{0}
$$

with $N_{0}$ defined by (1.14). 
We have $E_{-N}^{\Lambda}$ in (3.21) of order less than or equal to zero thanks to $N \geq N_{0}$ in (1.15). The problem (3.19) is well-posed in $H^{\infty}$ with a loss of $N_{0}$ derivatives.

Proof of Theorem 1.11. Under the Hölder continuity given by (1.16), the properties (3.8) and (3.10) become

$$
\begin{gathered}
\tilde{a}_{p+j} \in C\left([0, T] ; S^{0}\right) \cap C^{1}\left([0, T] ; S^{p-p \alpha_{p+j}}\right), \\
\tilde{b}_{j} \in C\left([0, T] ; S^{0}\right) \cap C^{1}\left([0, T] ; S^{p-p \beta_{j}}\right), \\
\tilde{a}_{p+j}-a_{p+j} \in C\left([0, T] ; S^{-p \alpha_{p+j}}\right), \tilde{b}_{j}-b_{j} \in C\left([0, T] ; S^{-p \beta_{j}}\right) .
\end{gathered}
$$

Consequently the factorization (3.2) becomes

$$
\begin{aligned}
L=L_{1}^{-}\left(t, x, D_{t}, D_{x}\right) L_{1}^{+}(t, x, & \left.D_{t}, D_{x}\right) \\
& +\sum_{j=1}^{p-1} R_{p+h-p \alpha_{p+j}}\left(t, x, D_{x}\right)+R_{h-p \beta_{j}}\left(t, x, D_{x}\right) D_{t}
\end{aligned}
$$

with $R_{q}$ of order $q$. Also here, as in the proof of Proposition 1.8, the operators $L_{1}^{ \pm}$ have real full symbols.

Now, the system $S$ in Lemma 3.2 is given by

$$
S=D_{t}+K+\sum_{j=1}^{p-1}\left(F_{j-p \alpha_{p+j}}+F_{h-p \beta_{j}}\right)+F_{0},
$$

where:

- the Hermitian part $\left(i K+(i K)^{*}\right) / 2$ of the diagonal operator $i K$ is of order $p-2$ and, from (1.17), with principal part $H_{K}^{0}$ which satisfies (3.23);

- the full matrices $F_{q}$ are of order $q$ for $q \geq 0$ with

$$
\left|F_{q}\right| \leq C\langle x\rangle^{-q /(p-1)}\langle\xi\rangle^{q}
$$

for $q>0$ from the decay assumptions in (1.16). For $p>2$, if there are orders $q>1$, then we also have

$$
\left|\partial_{x} F_{q}\right|+\left|\partial_{x x} F_{q}\right| \leq C\langle x\rangle^{-(q-1) /(p-1)}\langle\xi\rangle^{q}, q>1,
$$

from (1.18) and, eventually,

$$
\left|\partial_{x x x} F_{q}\right| \leq C\langle x\rangle^{-(q-1-1 / 2) /(p-1)}\langle\xi\rangle^{q}, q>1+1 / 2,
$$

from (1.19).

Also here from (1.9) + we have $\delta_{+}=\delta_{-}$in (3.20) and we may take $\delta_{+}=\delta_{-}=+1$ without loss of generality.

Let us take the symbol of order zero,

$$
\Lambda=\Lambda_{p-1-p \alpha_{2 p-1}}+\Lambda_{p-1-p \beta_{p-1}},
$$

where each $\Lambda_{q}$ is defined by (3.27) with a sufficiently large constant $M_{q}$. From (3.28), Theorem 2.2 and taking the order $p-2$ of $\left(i K+(i K)^{*}\right) / 2$ into account, there are an anti-Hermitian matrix operator $A_{p}$ of order $p$, positive matrix operators $P_{q}$ of order $q$ and a matrix operator $Q_{p-2}$ of order $p-2$ such that

$$
\left(e^{\Lambda}\right)^{-1} i S e^{\Lambda}=\partial_{t}+A_{p}+P_{p-1-p \alpha_{2 p-1}}+P_{p-1-p \beta_{p-1}}+Q_{p-2} .
$$


For $p=2$, this is already sufficient to obtain the well-posedness in $H^{s}$ of (3.19), hence of (1.1). For $p>2$, (3.23) and (2.5), together with (3.29) and (3.30), give

$$
\begin{aligned}
Q_{p-2} & =\sum_{q \in \mathcal{I}, q \geq 0} G_{q}, \\
\mathcal{I}=\left\{p-3, p-5 / 2-p \alpha_{2 p-1}, p-5 / 2-p \beta_{p-1}, p-2-p \alpha_{2 p-1},\right. & \\
& \left.p-2-p \beta_{p-1}, p-2-p \alpha_{2 p-2}, p-2-p \beta_{p-2}, p-2\right\},
\end{aligned}
$$

where each $G_{q}$ is of order $q$ and with symbol such that

$$
\left|G_{q}\right| \leq C\langle x\rangle^{-q /(p-1)}\langle\xi\rangle^{q}
$$

for $q>0$. Following (3.31), we add terms in the expansion of $\Lambda$, now taking

$$
\Lambda=\Lambda_{p-1-p \alpha_{2 p-1}}+\Lambda_{p-1-p \beta_{p-1}}+\sum_{q \in \mathcal{I}, q>0} \Lambda_{q}
$$

all the terms still defined by (3.27). We obtain

$$
\left(e^{\Lambda}\right)^{-1} i S e^{\Lambda}=\partial_{t}+A+P+Q_{p-3}
$$

with $A$ anti-Hermitian, $P$ positive and $Q_{p-3}$ of order $p-3$, concluding the proof for $p \leq 3$.

\section{REFERENCES}

[1] Cicognani, M.; Colombini, F.: Sharp regularity of the coefficients in the Cauchy Problem for a class of evolution equations. Differential and Integral Equations 16 (2003), 1321-1344. MR2016685 (2005a:35045)

[2] Cicognani, M.; Colombini, F.: Loss of Derivatives in Evolution Cauchy Problems. Ann. Univ. Ferrara 52 (2006), 271-280 (in memoria di Stefano Benvenuti). MR2273098(2008f:35039)

[3] Colombini, F.; De Giorgi, E.; Spagnolo, S.: Sur les équations hyperboliques avec des coefficients qui ne dependent que du temps. Ann. Sc. Norm. Sup. Pisa 6 (1979), 511-559. MR553796 (81c:35077)

[4] Colombini, F.; Lerner, N.: Hyperbolic operators with non-Lipschitz coefficients. Duke Math. J. 77 (1995), 657-698. MR.1324638 (96d:35075)

[5] Colombini, F.; Métivier, G.: The Cauchy Problem for Wave Equations with non Lipschitz Coefficients; Application to continuation of solutions of some nonlinear wave equations. Ann. Sci. École Norm. Sup. 41 (2008), 177-220. MR2468481(2009k:35194)

[6] Ichinose, W.: Some remarks on the Cauchy problem for Schrödinger type equations. Osaka J. Math. 21 (1984), 565-581. MR759481 (86f:35060)

[7] Kajitani, K.; Baba, A.: The Cauchy problem for Schrödinger type equations. Bull. Sci. Math. 119 (1995), 459-473. MR1354247 (96m:35060)

[8] Kumano-Go, H.: Pseudo-differential operators. The MIT Press, Cambridge, London, 1982.

[9] Mizohata, S.: On the Cauchy problem. Academic Press, Orlando, FL; Science Press, Beijing, 1985. MR860041 (89a:35007)

Facoltà di Ingegneria II, Via Genova, 181, 47023 Cesena, Italy

Current address: Dipartimento di Matematica, Piazza di Porta S. Donato, 5, 40127 Bologna, Italy

E-mail address: cicognani@dm.unibo.it

Dipartimento di Matematica, University of Pisa, Largo Bruno Pontecorvo, 5, 56127 Pisa, ITALY

E-mail address: colombini@dm.unipi.it 